\title{
Estimation of Aboveground Biomass For Winter Wheat In The Later Growth Stage By Combining Digital Texture And Spectral Analysis
}

Ling Zheng ( $\square$ lingz0865@163.com )

Anhui University https://orcid.org/0000-0002-2333-9555

\section{Tao Jianpeng}

Anhui University

\section{Bao Qian}

Anhui University

Weng Shizhuang

Anhui University

\section{Zhang Yakun}

Henan University of Science and Technology

Zhao Jinling

Anhui University

Huang Linsheng

Anhui University

\section{Research}

Keywords: aboveground biomass (AGB), wheat, canopy, vegetation indices, texture

Posted Date: December 21st, 2021

DOI: https://doi.org/10.21203/rs.3.rs-1132282/v1

License: (1) This work is licensed under a Creative Commons Attribution 4.0 International License. Read Full License 


\section{Abstract}

Background: Aboveground biomass (AGB) is an important indicator to predict crop yield. Traditional spectral features or image textures have been proposed to estimate the AGB of crops, but they perform poorly in estimation of AGB at high biomass levels. The present study thus evaluated the ability of spectral features, image textures, combinations thereof to estimate winter wheat AGB.

Result: The spectral features were obtained from the wheat canopy reflectance spectra of $400-1000 \mathrm{~nm}$ including original wavelengths and seven vegetation indices (VIs), then we screened effective wavelengths (EWs) through successive projection algorithm (SPA) and the optimal vegetation index selected by correlation analysis. The image textures features were extracted by gray level co-occurrence matrix including texture features (TEX) and normalized difference texture index (NDTI), then we selected effective variables including the optimal texture subset (OTEXS) and the optimal normalized difference texture index subset (ONDTIS) through the ranking of feature importance of random forest (RF). Linear regression (LR), partial least squares regression (PLS) and random forest (RF) were established to evaluate the relationship between each calculated feature and AGB. The results demonstrate that the ONDTIS with PLS based on validation datasets exhibited better performance in estimating AGB for the post-seedling stage $\left(R^{2}=0.75, R M S E=0.04\right)$. Moreover, the combinations of OTEXS and EWs with LR based on validation datasets exhibited the highest prediction accuracy for the post-seedling stage $\left(R^{2}=0.78, \mathrm{RMSE}=0.05\right)$.

Conclusion: The findings show that the combined use of spectral features and image textures can effectively improve the accuracy for AGB estimation especially in post-seeding stage.

\section{Introduction}

The aboveground biomass (AGB) is of great practical significance for monitoring crop growth [1] and predicting yield [2]. Therefore, the rapid and accurate prediction of AGB is critical to managing agricultural activities efficiently [3].

The conventional manual field measurement of AGB involves destructive sampling that is time-consuming and laborious [4]. Given these constraints, prompt and accurate monitoring of AGB is critical. Previous studies have demonstrated that multispectral or hyperspectral information from satellites or airborne platforms has been widely used for monitoring leaf area index [5], crop growth [6], nitrogen content [7], and biomass of wheat [8]. However, unfavourable weather conditions such as clouds or fog may lead to a lack of applicable satellite data, which limits the application of this data in crop monitoring. In particular, high temporal resolution is required to explain the spatial specificity in the field during the critical stage of crop monitoring [9]; the accuracy of phenological information needs to be determined from remote sensing observation, which depends largely on the frequency of observations [2]. At the same time, data from remote sensing satellites are usually expensive and require extensive data processing experience.

In recent years, with the development of unmanned aerial vehicles (UAVs) and their application in the field of remote sensing, the use of canopy spectra and UAV images has become a novel method for crop monitoring. For example, researchers have demonstrated the feasibility of using canopy spectra extracted from UAV hyperspectral images combined with partial least squares (PLS) regression to estimate the chlorophyll content of wheat [10]. In addition, colour index and crop surface models have been extracted by using orthogonal correction with ( red-green-blue) RGB UAV images to estimate a leaf area index (LAI) [11], plant height [12], and plant nitrogen content [13]. However, the spectral or image features obtained from a UAV are saturated in the later stage of crop growth, which leads to poor accuracy in the estimation of crop yield. In order to solve this problem, researchers have attempted to combine spectral and image features; they have found that the vegetation indices (VIs) combined with a textural feature index (a Normalized Differential Texture Index, NDTI) extracted from $550 \mathrm{~nm}$ and $800 \mathrm{~nm}$ band images obtained by UAV multispectral camera provided better results than using traditional textural features and vegetation indices in a rice AGB estimation model [14]. Previous studies have proved the feasibility of UAV-based textural features and VIs along with their combination in wheat AGB estimation and yield detection. However, due to the low resolution of the images obtained by most UAVs, the crop canopy information cannot be fully obtained in the case of large crop coverage in the later stage of growth, which leads to low accuracy in estimating wheat AGB in the later stage of growth [8]. The results show that the accurate estimation of crop AGB using the features obtained by a UAV platform needs to be improved in the later stage of crop growth.

Near-infrared spectroscopy is one of the common methods used to detect crop biomass [15]. Using a hand-held spectrometer to obtain crop canopy reflectance and then extracting a VI or effective wavelengths to estimate wheat biomass has been proven to be an effective method [16]. For example, a power function or exponential function relationship was found between a specific vegetation 
index (RVI) and AGB of soybean at the seedling stage [17]. However, when the crop biomass reaches a certain range, the crop canopy reflectance tends to be saturated, which leads to the low accuracy of estimated AGB in a crop model based on a VI. In order to reduce the effects of spectral saturation on crop AGB estimation, some researchers have used PLS regression based on band depth and a VI to estimate wheat biomass. However, the results show that the problem of canopy spectral saturation still exists [18]. However, image technology based on consumer grade digital cameras has been commonly used to monitor crop morphology [19], nutrient components [20], and pest status [21]. For example, image information can be obtained through a digital camera, and then the image can be processed into a 3D point cloud data for estimating wheat biomass, crown height, and a harvest index. Research shows that this measurement method has the advantages of high adaptability and robustness [22]. However, when plants grow to a certain level, the model used to estimate AGB based on image features also performs poorly. The results demonstrate that an estimation model based on individual spectral or image features cannot accurately estimate crop AGB in the later stage of growth.

Therefore, the purpose of this study is to evaluate the application of combining ground-scale images with spectral information in estimating the AGB of winter wheat. The image texture, spectral features, and their combinations are used to estimate the AGB of winter wheat in multiple growth stages. In this study, several methods that can be used to predict the AGB of winter wheat are proposed that are based on: (1) spectral features (VIs, effective wavelength), (2) the OTEXS and the ONDTIS calculated from canopy images and side images, and (3) the combination features with the random forest (RF) regression and PLS regression.

\section{Results}

\section{Estimation of AGB with Effective Wavelengths}

The near infrared (400-1000 $\mathrm{nm}$ ) reflectance spectra of the winter wheat canopy was measured. In order to eliminate the random error and scattering effect, the SG and MSC were selected to preprocess the spectrum; it can be seen that the interference of the treated spectral curve was obviously reduced (Figure 4). Meanwhile, note that the adjacent wavelengths were usually highly correlated. In order to reduce information redundancy and improve the utilization efficiency of spectral information, a successive projection algorithm (SPA) was used to select the effective wavelengths of the processed spectrum.

Figure 5 presents the results of effective wavelength selection using an SPA model at the seedling stage. The RMSE values of different subsets in the SPA model during seedling stage are shown in Figure $5 a$, where " $\square$ " represents the number of the effective wavelength. The results show that when the number of variables was less than four, the RMSE value shows a downward trend. In contrast, the change tends to be flat. Therefore, four effective wavelengths were extracted by SPA at seedling stage to estimate the AGB of winter wheat. Figure $5 b$ shows the specific selection of effective wavelengths at the seedling stage, in which " $\square$ " represents the selected effective wavelengths ( $583.35 \mathrm{~nm}, 762.553 \mathrm{~nm}, 929.176 \mathrm{~nm}$, and $940.639 \mathrm{~nm})$. Similarly, the number of effective wavelengths selected by the SPA at the post-seedling and all stages were 12 and 14 , respectively.

The results of the model that predicts the AGB of winter wheat based on the effective wavelengths (Table 3 ) at multiple growth stages. The results show that the LR model based on effective wavelength estimation of winter wheat AGB had a higher $\mathrm{R}^{2}$ and lower RMSE at the seedling stage $\left(R^{2}=0.89\right.$, RMSE $\left.=0.016\right)$, the post-seedling $\left(R^{2}=0.73\right.$, RMSE $\left.=0.07\right)$, and in all stage $\left(R^{2}=0.83, R M S E=0.06\right)$ (Figure 6).

Table 3. Aboveground biomass estimates using selected effective wavelengths in different growth stages.

\begin{tabular}{|c|c|c|c|c|c|c|c|c|c|c|c|c|}
\hline & LR & & & & RF & & & & PLS & & & \\
\hline & $\mathrm{R}_{c}^{2}$ & $\mathrm{RMSE}_{c}$ & $\mathrm{R}_{v}^{2}$ & $\mathrm{RMSE}_{v}$ & $\mathrm{R}_{c}^{2}$ & $\mathrm{RMSE}_{c}$ & $\mathrm{R}_{v}^{2}$ & $\mathrm{RMSE}_{v}$ & $\mathrm{R}_{c}^{2}$ & $\mathrm{RMSE}_{c}$ & $\mathrm{R}_{v}^{2}$ & $\mathrm{RMSE}_{v}$ \\
\hline Seedling & 0.89 & 0.01 & 0.89 & 0.01 & 0.96 & 0.01 & 0.84 & 0.02 & 0.91 & 0.01 & 0.89 & 0.01 \\
\hline Post-Seedling & 0.72 & 0.06 & 0.73 & 0.07 & 0.92 & 0.03 & 0.69 & 0.06 & 0.74 & 0.05 & 0.67 & 0.05 \\
\hline All & 0.84 & 0.06 & 0.83 & 0.06 & 0.95 & 0.03 & 0.72 & 0.07 & 0.86 & 0.05 & 0.83 & 0.06 \\
\hline
\end{tabular}




\section{Estimation of AGB with VIs}

Figure 7 shows that a significant positive correlation existed between VIs and winter wheat AGB. The AGB of winter wheat was predicted well by VIs with LR, RF, PLS models in the seedling stage and when all stages were considered together, but the prediction accuracy of winter wheat AGB in the post-seedling stage was lower (Table 4). Among them, the LR prediction model based on a renormalized difference vegetation index had the best prediction ability to estimate the winter wheat AGB at the seedling stage $\left(R^{2}=\right.$ 0.82 , RMSE $=0.02$ ). In the all stages, the estimation of AGB by the LR model based on an optimized soil adjusted vegetation index had a good prediction effect $\left(R^{2}=0.68\right.$, RMSE $\left.=0.09\right)$. However, with the continuous growth of winter wheat, the correlation between each individual VI and winter wheat AGB was low in the post-seedling stage (Figure 8). The accuracy of predictive model of winter wheat AGB based on each individual vegetation index separately performed poorly, of which the LR model based on an enhanced vegetation index had the best predictability $\left(R^{2}=0.45, R M S E=0.1\right)$. The results show that the $L R$ model based on a single vegetation index cannot accurately estimate the AGB of winter wheat in the post-seedling stage.

Table 4

Prediction results of LR, RF, PLS with the optimal VI across all different growth stages.

\begin{tabular}{|c|c|c|c|c|c|c|c|c|c|c|c|c|c|}
\hline \multirow[t]{2}{*}{ Stage } & \multirow[t]{2}{*}{ VI } & \multicolumn{4}{|l|}{ LR } & \multicolumn{4}{|l|}{ RF } & \multicolumn{4}{|l|}{ PLS } \\
\hline & & $\mathrm{R}_{c}^{2}$ & $\mathrm{RMSE}_{c}$ & $\mathrm{R}_{v}^{2}$ & $\mathrm{RMSE}_{v}$ & $\mathrm{R}_{c}^{2}$ & $\mathrm{RMSE}_{c}$ & $\mathrm{R}_{v}^{2}$ & $\mathrm{RMSE}_{v}$ & $\mathrm{R}_{c}^{2}$ & $\mathrm{RMSE}_{c}$ & $\mathrm{R}_{v}^{2}$ & $\mathrm{RMSE}_{v}$ \\
\hline Seedling & RDVI & 0.83 & 0.02 & 0.8 & 0.02 & 0.94 & 0.01 & 0.82 & 0.02 & 0.9 & 0.01 & 0.82 & 0.02 \\
\hline $\begin{array}{l}\text { Post- } \\
\text { Seedling }\end{array}$ & EVI & 0.45 & 0.08 & 0.45 & 0.1 & 0.89 & 0.04 & 0.4 & 0.09 & 0.48 & 0.06 & 0.43 & 0.05 \\
\hline All & OSAVI & 0.68 & 0.08 & 0.67 & 0.08 & 0.93 & 0.03 & 0.68 & 0.09 & 0.7 & 0.07 & 0.63 & 0.07 \\
\hline
\end{tabular}

\section{Estimation of AGB with textural feature}

The relationship between textural features of the $\mathrm{R}, \mathrm{G}$, and $\mathrm{B}$ bands along with the AGB of winter wheat varied greatly at multiple growth stages (Table 5). Most single textures had poor prediction performance for estimating AGB at all stages. In the seedling stage, the textural features of R, G, and B bands (mean, variation, Con, Dis) were significantly correlated with AGB. In the post-sowing stage, the correlation between the textural features of the three bands (Hom, Ent and Sem) and winter wheat AGB decreased significantly. Therefore, a single textural feature cannot predict AGB comprehensively and accurately, which was consistent with using a single vegetation index to predict the AGB of winter wheat. 
Table 5

Relationships between above-ground biomass and grey level co-occurrence matrix-based texture measurements with the calibration $\operatorname{set}\left(R^{2}\right)$.

\begin{tabular}{|llllllllll|}
\hline & Red band & & \multicolumn{3}{c|}{ Green band } & \multicolumn{5}{c|}{ Blue band } \\
\hline & Seedling & Post-Seedling & All & Seedling & Post-Seedling & All & Seedling & Post-Seedling & All \\
\hline Mean & 0.736 & 0.392 & $\mathbf{0 . 3 8 4}$ & 0.715 & 0.326 & $\mathbf{0 . 2 4}$ & $\mathbf{0 . 7 2 5}$ & 0.509 & 0.02 \\
\hline Var & 0.787 & 0.017 & 0.004 & 0.685 & 0.033 & 0.004 & 0.603 & 0.022 & 0.095 \\
\hline Hom & 0.567 & 0.562 & 0.074 & 0.559 & $\mathbf{0 . 5 8 2}$ & 0.001 & 0.56 & 0.599 & 0.262 \\
\hline Con & 0.787 & 0.014 & 0.017 & 0.674 & 0.026 & 0.008 & 0.6 & 0.02 & 0.042 \\
\hline Dis & $\mathbf{0 . 7 9 1}$ & 0.253 & 0.001 & $\mathbf{0 . 7 1 7}$ & 0.308 & 0.009 & 0.715 & 0.314 & 0.169 \\
\hline Ent & 0.236 & $\mathbf{0 . 5 7 2}$ & 0.013 & 0.233 & 0.59 & 0.001 & 0.361 & $\mathbf{0 . 6 0 2}$ & 0.091 \\
\hline Sem & 0.04 & 0.555 & 0.017 & 0.033 & 0.577 & 0.001 & 0.152 & 0.575 & 0.076 \\
\hline Cor & 0.005 & 0.006 & 0.004 & 0.001 & 0.004 & 0.191 & 0.019 & 0.004 & 0.017 \\
\hline
\end{tabular}

From the above results, one can see that the LR model based on only a single textural feature performed well in the seedling stage; the correlation between most textural features and winter wheat AGB was not significant during the post-seedling and all combined stages. Therefore, in order to improve the ability of the LR model to estimate wheat AGB based on a single textural feature, this paper proposes to the optimal textural feature subset (OTEXS) for use as the input for the winter wheat AGB prediction model. we employed a RF model to calculate the texture features importance as well as to sort and the best textural features were selected to form the optimal textural feature subset, the number of OTEXS in the different growth stages was six.

When compared with the prediction model of winter wheat AGB based on texture features, the estimation accuracy of LR, RF, PLS prediction model based on OTEXS was significantly improved in the post-seedling and all stages (Table 6). The accuracy of the OTEXSbased RF model was significantly improved in the post-seedling stage $\left(R^{2}=0.73\right.$, RMSE $\left.=0.07\right)$ and the all stages $\left(R^{2}=0.75\right.$, RMSE $=$ 0.06). The LR model of AGB based on OTEXS also provided the great estimation performance at the seedling stage $\left(R^{2}=0.85, R M S E=\right.$ 0.01). The results show that OTEXS was one of the promising schemes that can be used to improve the prediction accuracy of an LR model for estimating AGB at multiple growth stages (Figure 9).

Table 6

Prediction results of LR, RF, PLS with the optimal texture subset across all different growth stages.

\begin{tabular}{|c|c|c|c|c|c|c|c|c|c|c|c|c|}
\hline & \multicolumn{4}{|l|}{ LR } & \multicolumn{3}{|l|}{$\mathrm{RF}$} & \multicolumn{2}{|c|}{ PLS } & \multirow[b]{2}{*}{$\mathrm{RMSE}_{c}$} & \multirow[b]{2}{*}{$\mathrm{R}_{v}^{2}$} & \multirow[b]{2}{*}{$\mathrm{RMSE}_{v}$} \\
\hline & $\mathrm{R}_{c}^{2}$ & $\mathrm{RMSE}_{c}$ & $\mathrm{R}_{v}^{2}$ & $\mathrm{RMSE}_{v}$ & $\mathrm{R}_{c}^{2}$ & $\mathrm{RMSE}_{c}$ & $\mathrm{R}_{v}^{2}$ & $\mathrm{RMSE}_{v}$ & $\mathrm{R}_{c}^{2}$ & & & \\
\hline Seedling & 0.90 & 0.01 & 0.85 & 0.01 & 0.96 & 0.01 & 0.83 & 0.02 & 0.91 & 0.01 & 0.85 & 0.01 \\
\hline $\begin{array}{l}\text { Post- } \\
\text { Seedling }\end{array}$ & 0.76 & 0.06 & 0.66 & 0.07 & 0.93 & 0.03 & 0.73 & 0.07 & 0.77 & 0.05 & 0.65 & 0.06 \\
\hline All & 0.81 & 0.06 & 0.76 & 0.06 & 0.94 & 0.03 & 0.75 & 0.06 & 0.82 & 0.05 & 0.77 & 0.07 \\
\hline
\end{tabular}

In the present study, the researchers attempted to combine two different textures randomly to construct a new texture parameter NDTI for AGB estimation [7]. Figure 10 shows that the $R^{2}$ of LR models based on NDTI for estimating AGB has significant difference at multiple growth stages. When compared with the other two growth stages, the LR model based on NDTI performed best for winter wheat AGB estimation at the seedling stage, in which the NDTI (Rmean, Gmean) had the best performance with $\mathrm{R}^{2}$ of 0.94 . In the postseedling and all stages, the NDTI-based LR model also achieved better performance in the estimation of AGB than a single textural 
feature. That is, NDTI (Bean, Bhom) performed best with an $\mathrm{R}^{2}$ of 0.67 at the post-seedling stage; the NDTI (Ghom, Bhom) performed the best with $\mathrm{R}^{2}$ of 0.51 at the all stage. Table 7 shows the top five best performing NDTIs at multiple growth stages (Figure 11).

Table 7

Performance of the top-five normalized differential texture index-based linear regression models based on calibration dataset.

\begin{tabular}{|c|c|c|c|c|c|c|c|c|}
\hline \multicolumn{3}{|l|}{ Seedling } & \multicolumn{3}{|c|}{ Post-seedling } & \multicolumn{3}{|l|}{ All } \\
\hline $\mathrm{x} 1$ & $x 2$ & $\mathrm{R}^{2}$ & $\mathrm{x} 1$ & $x 2$ & $\mathrm{R}^{2}$ & $\mathrm{x} 1$ & $x 2$ & $\mathrm{R}^{2}$ \\
\hline Rmean & Rcor & 0.93 & Bmean & Bent & 0.67 & Ghom & Bhom & 0.51 \\
\hline Rmean & Gcor & 0.9 & Gent & Bmean & 0.64 & Rmean & Rcor & 0.48 \\
\hline Rmean & Bdis & 0.86 & Rent & Gcor & 0.64 & Rcon & Gcon & 0.48 \\
\hline Rmean & Gdis & 0.85 & Gmean & Bent & 0.62 & Rhom & Bhom & 0.45 \\
\hline Rdis & Gent & 0.84 & Gmean & Gent & 0.62 & Rdis & Bdis & 0.45 \\
\hline
\end{tabular}

The conclusion shows that the low accuracy of the AGB prediction model based on a single NDTI also exists in the post-seedling stage and all stage. Therefore, this paper employed a RF model to calculate the feature importance as well as to sort and select the NDTI with highest importance as the optimal NDTI subset (ONDTIS) to estimate the AGB with the LR, RF, PLS model. The number of NDTIs of ONDTIS in each growth stage were 8,13 and 12, respectively. Compared with an individual NDTI, the PLS models of AGB based on the ONDTIS were improved in multiple stages (Table 8). In the seedling stage, the ONDTIS preformed best with the highest $R^{2}=0.91$ and lowest RMSE $=0.01$. Excellent results were also obtained in the post-seedling and the all stages.

Table 8

Prediction results of LR, RF, PLS with the optimal normalized difference texture index subset across all different growth stages.

\begin{tabular}{|c|c|c|c|c|c|c|c|c|c|c|c|c|}
\hline \multicolumn{3}{|c|}{ LR } & \multicolumn{4}{|c|}{ RF } & \multicolumn{4}{|c|}{ PLS } & \multirow[b]{2}{*}{$\mathrm{R}_{v}^{2}$} & \multirow[b]{2}{*}{$\mathrm{RMSE}_{v}$} \\
\hline & $\mathrm{R}_{c}^{2}$ & $\mathrm{RMSE}_{c}$ & $\mathrm{R}_{v}^{2}$ & $\mathrm{RMSE}_{v}$ & $\mathrm{R}_{c}^{2}$ & $\mathrm{RMSE}_{c}$ & $\mathrm{R}_{v}^{2}$ & $\mathrm{RMSE}_{v}$ & $\mathrm{R}_{c}^{2}$ & $\mathrm{RMSE}_{c}$ & & \\
\hline Seedling & 0.95 & 0.01 & 0.86 & 0.019 & 0.95 & 0.01 & 0.82 & 0.02 & 0.93 & 0.01 & 0.91 & 0.01 \\
\hline $\begin{array}{l}\text { Post- } \\
\text { Seedling }\end{array}$ & 0.82 & 0.05 & 0.74 & 0.07 & 0.94 & 0.02 & 0.73 & 0.06 & 0.78 & 0.05 & 0.75 & 0.04 \\
\hline All & 0.82 & 0.066 & 0.77 & 0.07 & 0.94 & 0.03 & 0.82 & 0.06 & 0.82 & 0.05 & 0.78 & 0.06 \\
\hline
\end{tabular}

\section{Estimation of winter wheat AGB with combination features}

The winter wheat AGB prediction models based on a single spectral or image feature in the post-seedling and the all stages had limitations and the low accuracy. Therefore, a multiple regression prediction model of winter wheat AGB was established by combining the spectral and image information. This provided appropriate technical support for improving the estimation accuracy of winter wheat AGB in the post-seeding stage. The combination feature was formed by the combination of effective wavelengths and VI, OTEXS and ONDTIS. The results of estimating winter wheat AGB based on combination features at multiple growth stages are shown in Table 9. The PLS regression estimation model of AGB based on the combination of OTEXS and effective wavelength had the highest accuracy at the seedling $\left(R^{2}=0.943\right.$, RMSE $\left.=0.01\right)$, and the LR estimation model of AGB based on the combination of OTEXS and effective wavelength had the highest accuracy at post-seedling stages $\left(R^{2}=0.78, R M S E=0.06\right)$, Figure 12. The RF-based winter wheat $A G B$ prediction model based on the combination of OTEXS and effective wavelength also had the highest accuracy in the all stage $\left(R^{2}=\right.$ 0.87 , RMSE $=0.05$ ). Figure 13 reveals the accuracy assessment results of the multivariate regression models for AGB estimation based on the independent validation datasets. As shown in Figure 13a-i, for the three regression methods ( $L R, R F$ and PLS), the accuracy of 
the models based on combined features was better than that based on image textures or spectral features for estimating winter wheat AGB, especially in the post-seedling stage.

Table 9

Prediction results of LR, RF, PLS with the combination features across all different growth stages.

\begin{tabular}{|c|c|c|c|c|c|c|c|c|c|c|c|c|c|}
\hline \multirow[t]{2}{*}{ Stage } & \multirow[t]{2}{*}{ Features } & \multicolumn{4}{|l|}{ LR } & \multicolumn{4}{|l|}{ PLS } & \multicolumn{4}{|l|}{ RF } \\
\hline & & $\mathrm{R}_{c}^{2}$ & $\mathrm{RMSE}_{c}$ & $\mathrm{R}_{v}^{2}$ & $\mathrm{RMSE}_{v}$ & $\mathrm{R}_{c}^{2}$ & $\mathrm{RMSE}_{c}$ & $\mathrm{R}_{v}^{2}$ & $\mathrm{RMSE}_{v}$ & $\mathrm{R}_{c}^{2}$ & $\mathrm{RMSE}_{c}$ & $\mathrm{R}_{v}^{2}$ & $\mathrm{RMSE}_{v}$ \\
\hline \multirow[t]{4}{*}{ Seedling } & VI+OTEXS & 0.95 & 0.01 & 0.85 & 0.02 & 0.93 & 0.01 & 0.92 & 0.01 & 0.97 & 0.007 & 0.88 & 0.02 \\
\hline & $\begin{array}{l}\text { EWs + } \\
\text { OTEXS }\end{array}$ & 0.98 & 0.01 & 0.85 & 0.02 & 0.96 & 0.009 & 0.94 & 0.01 & 0.97 & 0.008 & 0.82 & 0.02 \\
\hline & $\begin{array}{l}\text { ONDTIS + } \\
\text { EWs }\end{array}$ & 0.96 & 0.01 & 0.94 & 0.01 & 0.97 & 0.007 & 0.89 & 0.01 & 0.97 & 0.007 & 0.77 & 0.02 \\
\hline & $\begin{array}{l}\text { ONDTIS + } \\
\text { VI }\end{array}$ & 0.95 & 0.01 & 0.87 & 0.01 & 0.93 & 0.01 & 0.91 & 0.01 & 0.97 & 0.007 & 0.77 & 0.02 \\
\hline \multirow{4}{*}{$\begin{array}{l}\text { Post- } \\
\text { Seedling }\end{array}$} & VI+OTEXS & 0.77 & 0.06 & 0.72 & 0.06 & 0.80 & 0.05 & 0.71 & 0.06 & 0.95 & 0.02 & 0.70 & 0.06 \\
\hline & $\begin{array}{l}\text { EWs + } \\
\text { OTEXS }\end{array}$ & 0.82 & 0.05 & 0.78 & 0.05 & 0.84 & 0.04 & 0.76 & 0.05 & 0.96 & 0.02 & 0.74 & 0.06 \\
\hline & $\begin{array}{l}\text { ONDTIS + } \\
\text { EWs }\end{array}$ & 0.82 & 0.05 & 0.8 & 0.05 & 0.83 & 0.04 & 0.71 & 0.06 & 0.96 & 0.02 & 0.77 & 0.05 \\
\hline & $\begin{array}{l}\text { ONDTIS + } \\
\text { VI }\end{array}$ & 0.8 & 0.05 & 0.74 & 0.06 & 0.80 & 0.05 & 0.74 & 0.05 & 0.95 & 0.02 & 0.72 & 0.06 \\
\hline \multirow[t]{4}{*}{ ALL } & VI+OTEXS & 0.85 & 0.06 & 0.81 & 0.05 & 0.85 & 0.05 & 0.83 & 0.06 & 0.97 & 0.02 & 0.86 & 0.05 \\
\hline & $\begin{array}{l}\text { EWs + } \\
\text { OTEXS }\end{array}$ & 0.88 & 0.05 & 0.85 & 0.05 & 0.89 & 0.04 & 0.83 & 0.06 & 0.97 & 0.02 & 0.87 & 0.05 \\
\hline & $\begin{array}{l}\text { ONDTIS + } \\
\text { EWs }\end{array}$ & 0.88 & 0.05 & 0.87 & 0.05 & 0.89 & 0.04 & 0.82 & 0.06 & 0.97 & 0.02 & 0.85 & 0.05 \\
\hline & $\begin{array}{l}\text { ONDTIS + } \\
\text { VI }\end{array}$ & 0.87 & 0.05 & 0.77 & 0.06 & 0.85 & 0.05 & 0.83 & 0.05 & 0.97 & 0.02 & 0.85 & 0.05 \\
\hline
\end{tabular}

\section{Discussion}

\section{Estimation of AGB with spectral and texture feature}

Spectral techniques have been widely used to predict crop biomass and yield [24]. This paper shows that the prediction accuracy of the AGB with LR model based on effective wavelengths in the post-seedling stage $\left(R^{2}=0.73\right.$, RMSE $\left.=0.07\right)$ was improved when compared with results based on a vegetation index alone $\left(R^{2}=0.45\right.$, RMSE $\left.=0.1\right)$. The reason is that the wavelength selected by SPA includes the red (550-770 nm) and near-infrared regions (800-1000 nm), which are the main absorption bands of plant photosynthesis after sowing as seedling mature, while VI only uses the spectral information of several bands. Note that when compared with the AGB model at the seedling stage $\left(R^{2}=0.89, \mathrm{RMSE}=0.01\right)$, the accuracy of the $A G B$ prediction model had poor performance $\left(R^{2}=0.73, R M S E=\right.$ 0.07 ) in the post-seedling stage, which is similar to the model based on VI. The reason is that with the maturity of the canopy, the increase of leaf layer led to an increase of canopy complexity so that shadow became a spectral trap of incident energy and reduced the amount of radiation returned to the sensor [28]. It can be seen that AGB cannot be comprehensively and accurately predicted based on a single spectral feature. 
In recent years, researchers have found that image texture features can improve the effect of using satellite images to predict forest biomass [29]. In this paper, the correlation between a single textural feature and AGB was quite different in multiple stages which was consistent with the discovery reported in this reference [30]. The AGB prediction model based on the single textural feature (mean and dis) at the seedling stage resulted in the best $R^{2}$ (Table 5). The reason was that the canopy of winter wheat had rich structural information at the seedling stage. The mean and dis contain the average value in the moving window of the target and the background, which can smooth an image and minimize the background interference [7]. In this article, most of the textural features have poor correlations with the AGB of winter wheat. This result provided a similar conclusion found in the correlation between textural features and the AGB in temperate forests [31]. By aiming at the poor correlation between textural features and AGB, it was found that the NDTIbased rice AGB prediction model had a great performance at multiple stages [7]. A total of 276 NDTIs have been calculated in this paper; as a result, the correlation between NDTI and AGB was found to be different in multiple stages. Compared with textural features, the correlation between most NDTIs and AGB was obviously improved in multiple stages, while the accuracy of the AGB prediction model based on these NDTIs was better than that of an AGB model based on textural features. Among them, the AGB prediction model based on NDTI (Rmean and Rcon) in the seedling stage had the optimal effect $\left(R^{2}=0.93\right)$. The correlation between NDTI and AGB in the seedling stage was better than that in the late and all stages, which was consistent with the results of the analysis of textural features. The occurred because the canopy structure information was most accurately captured by digital images in the seedling stage in which the density of plants was low and the adjacent leaves hardly overlap. However, with the continuous growth of winter wheat, the canopy leaves of each plant overlap with each other, which resulted in a loss of structural information in digital images and decreased the accuracy of the winter wheat AGB prediction model based on image features. This result is consistent with the previous studies that used convolution neural networks to predict biomass-related shapes. The above results show that the AGB cannot be predicted comprehensively and accurately by using a single image feature.

Compared with the models based on a single spectral feature or single image feature, it was found that the AGB model based on the effective wavelength of a single spectral feature was better than a model based on a single image feature in seedling and all stages; however, in the post-seedling stage the accuracy of the AGB model based on ONDTIS was better than the spectral feature model. [32]found that a large amount of spectral information is lost in the later stages of crop growth, which makes it difficult to accurately predict yield. Therefore, the image and spectral information sources have different effects on the AGB prediction model in multiple growth stages. At the seedling stage, due to the simple three-dimensional structure of the crop canopy, the spectral and image information has a strong correlation with AGB. In contrast, the correlation between the spectral reflectance of the canopy and the AGB of winter wheat decreases because of the leaves obstruct each other leading to a loss of canopy information during the growth of the crop canopy.

\section{Estimation of AGB with optimal image features}

In view of the poor prediction performance of the AGB model based on a single textural feature and single NDTI, this paper proposes to fuse all textural features of the R, G, and B bands and all NDTIs for use as the input for the winter wheat AGB prediction model. However, considering that a large number of image features are mixed with more redundant information, this may reduce the accuracy of the LR model in estimating AGB; therefore, the important coefficients of all image features were calculated and sorted, its main purpose is to eliminate irrelevant or redundant features, save space and reduce calculation costs [33]. In this paper, RF was selected to extract data features and rank the importance of features, so that we can determine the contribution of each feature to each tree in the RF, and select image features whose importance was no less than $10 \%$ and $40 \%$ of the highest importance as the OTEXS and ONDTIS, respectively. The best texture features and NDTIs were selected to form the OTEXS and NDTIS which were used as the input of the LR model to estimate the wheat AGB. The results show that the performance of the AGB model based on the optimal subset of image features was improved in the post-seedling stage and in the all stages.

\section{Comparison of regression methods at multiple growth stages}

The PLS regression and RF were selected to establish the AGB prediction model based on the fusion data. Note that the prediction model based on the combination of spectral and image features (OTEXS + EWs) had better prediction performance than the spectral feature combinations (vegetation index and feature Effective wavelength) and image feature combinations (OTEXS and ONDTIS) (Table 9). The results show that the combination of image and spectral information has richer information. At the same time, the RF model was found to perform better in the post-seedling stage. This occurred because the RF regression model will build a large number of decision trees in the training process of dealing with a large number of samples, which has a good performance in dealing with outliers and noise. 


\section{Conclusions}

This study shows that the prediction accuracy of winter wheat AGB can be improved by using a combination feature analysis method. In order to evaluate the contribution of image and spectral features and their combination to predict AGB, the LR, PLS and RF were used to evaluate the ability of the combination to estimate winter wheat AGB based on the combined feature parameters (OTEXS+VI,

OTEXS+EWs, EWs + ONDTI and VI+ONDTI). Due to the canopy structure information was most accurately captured by digital images, It has a great advantage in predicting wheat AGB. The LR, PLS and RF model based on the combination of OTEXS from image features and EWs from spectral features had the highest accuracy in estimating winter wheat $A G B$ in the post-seedling stage $\left(R^{2}=0.788\right.$ and RMSE $=0.059$ ). The results show that the feature selection has great potential to improve the ability of researchers to estimate winter wheat AGB when compared with tradition textural features in the post-seedling stage. This method has a certain reference value for estimating crop AGB in the post-seeding stage. In the future work, more spatial effectives of wheat and analysis models will be used to develop a reliable crop AGB estimation model.

\section{Methods}

\section{Field Experiment and Measurements of Above-Ground Biomass}

The experiment in the present study was conducted in the Demonstration Base of National Precision Agriculture located in Xiaotangshan Town, Changping District $\left(40^{\circ} 00^{\prime}-40^{\circ} 21^{\prime} \mathrm{N}, 116^{\circ} 34^{\prime}-117^{\circ} 00^{\prime} \mathrm{E}\right)$, Beijing, China (Figure 1). Ten wheat varieties, Wanmai 38 , Zhongmai 11, Lunxuan 518, and other seven kinds were adopted in the experiment which ran from October 2013 to June 2014. Each variety was transplanted at two planting densities, $330 \mathrm{~kg} / \mathrm{hm} 2$ and $165 \mathrm{~kg} / \mathrm{hm} 2$. In this study, 40 plots, each with an area of $0.5 \mathrm{~m} \times$ $0.6 \mathrm{~m}$, were selected from 40 sample plots as experimental objects. A total of five planting periods were selected for field experiments (seedling, jointing, heading, flowering, and filling stages).

Ground destructive samplings were used to harvest the wheat in each measurement area on the ground. The fresh weight of each sample was measured by an electronic scale with an accuracy of $0.5 \mathrm{~g}$ and a range of $5 \mathrm{~kg}$. Altogether, 200 winter wheat AGB samples were obtained (40 samples in each of the five stages listed above). Table 1 shows the statistical data for measurement of winter wheat AGB.

Table 1

Descriptive statistics of above-ground biomass measurements in different growth stages.

\begin{tabular}{|lllllll|}
\hline Dataset & Stage & Samples & Max/kg & Mean/kg & Min/kg & Std/kg \\
\hline Calibration & Seedling & 27 & 0.23 & 0.102 & 0.04 & 0.05 \\
& Post-Seedling & 107 & 0.73 & 0.37 & 0.08 & 0.13 \\
\cline { 2 - 3 } & All & 134 & 0.73 & 0.317 & 0.04 & 0.16 \\
\hline \multirow{2}{*}{ Validation } & Seedling & 13 & 0.232 & 0.106 & 0.047 & 0.054 \\
& Post-Seedling & 53 & 0.641 & 0.372 & 0.121 & 0.125 \\
\hline & All & 66 & 0.639 & 0.317 & 0.047 & 0.156 \\
\hline
\end{tabular}

\section{Digital Image and Spectrum Data Acquisition}

Two different types of data were collected during two different time periods as described below. The image data of each wheat canopy sample were acquired using a UC-M3151 industrial camera (MicroVision, The China) with an image resolution of 3 megapixels at the seedling stage. Photos were acquired on clear cloudless or partly cloudy days; the specific time was from 6:00-8:00, Beijing time. In order to ensure the consistency of shooting angle and height of multiple lenses, a white rectangular frame with an area of $0.5 \mathrm{~m} \times 0.6$ $\mathrm{m}$ and a tripod with adjustable height were built as auxiliary devices for field image acquisition; next, the tripod was adjusted to make the photos perpendicular to the wheat canopy and $1 \mathrm{~m}$ from the canopy at the seedling stage. In the post-seedling stages (jointing to filling stages), the height of wheat plants increased continuously, the wheat leaves between different rows began to overlap with each other, and the visual field of the canopy image that was captured was almost filled with wheat leaves; this led to little change being visible in the proportion of wheat in the canopy in the background image. Therefore, the winter wheat AGB was predicted by taking single-row side images of wheat and extracting textural features. The side image acquisition auxiliary device included a white 
background plate with an area of $1.2 \mathrm{~m} \times 1.2 \mathrm{~m}$ and a tripod with adjustable height. While taking photographs of winter wheat, a white background plate was placed parallel to a row of wheat on the outside of the marking area and stood vertically on the ground (Figure 2). The tripod height was adjusted to about $0.5 \mathrm{~m}$; the lens was parallel to the white back plate while the distance from the side of the wheat was about $1.2 \mathrm{~m}$.

An AvaSpec-2048×14 fibre optic spectrometer (Avantes, Apeldoorn, The Netherlands) was used to collect spectral reflectance data with a wavelength range of $200-1100 \mathrm{~nm}$ and a spectral resolution was $2.4 \mathrm{~nm}$. The spectral reflectance experiments were conducted in clear cloudless or partly cloudy days; the specific time was from 10:30 to 14:30 (Beijing time). When collecting the spectral data, the optical fibre probe was about $0.75 \mathrm{~m}$ vertically below the wheat leaf canopy; standard reference plate correction was performed every $15 \mathrm{~min}$. Each plot was repeatedly measured five times and the average value was regarded as the spectrum measurement result for each plot.

\section{Data Processing}

\section{Spectral Data Processing}

Because the reflectance of the canopy experienced significant interference from the reflectance of the $200-400 \mathrm{~nm}$ and $1000-1100 \mathrm{~nm}$ bands, the canopy spectral reflectance of 400-1000 nm was selected for use in measuring the effective spectral parameters. SavitzkyGolay (S-G) smoothing can reduce the influence of high-frequency noise on the spectrum by averaging the multi-point spectral data; in addition, multiplicative scattering correction (MSC) can effectively eliminate the spectral difference which was caused by the different scattering levels of different spectral bands. A combination of MSC and S-G smoothing was used to preprocess the spectral data to reduce the influence of background noise and spectral scattering, thereby enhancing the correlation between the spectrum and the winter wheat AGB.

\section{Vegetation Indices Calculation}

In this study, six commonly-used VIs (Table2) were selected to evaluate the capability of spectral information for AGB estimation. The selected Vls were based on bands at $440,680,750$, and $810 \mathrm{~nm}$ of canopy reflectance.

Table2. Selected vegetation indices in this study for aboveground biomass estimation.

\begin{tabular}{|lll|}
\hline $\begin{array}{l}\text { Vegetation } \\
\text { Index }\end{array}$ & Formula & Reference \\
\hline OSAVI & $(1+0.16)\left(R_{810}-R_{750}\right) /\left(R_{810}-R_{750}+0.16\right)$ & {$[34]$} \\
\hline NDVI & $\left(R_{810}-R_{680}\right) /\left(R_{810}-R_{680}\right)$ & {$[35]$} \\
\hline DATT & $\left(R_{810}-R_{720}\right) /\left(R_{810}-R_{680}\right)$ & {$[36]$} \\
\hline $\begin{array}{l}\text { Cl red } \\
\text { edge }\end{array}$ & $\left(R_{810} / R_{750}\right)-1$ & {$[37]$} \\
\hline EVI & $2.5\left(R_{810}-R_{750}\right) /\left(1+R_{810}+6 R_{750}-7.5 R_{440}\right)$ & {$[38]$} \\
\hline RDVI & $\left(R_{810}-R_{750}\right) / \sqrt{R_{810}+R_{750}}$ & {$[39]$} \\
\hline $\begin{array}{l}\text { Note: OSAVI, optimized soil adjusted vegetation index; NDVI, normalized difference vegetation index; DATT; Cl red edge, red edge } \\
\text { chlorophyll index; EVI, enhanced vegetation index; } R D V I, \text { renormalized difference vegetation index }\end{array}$ \\
\hline
\end{tabular}

\section{Texture Measurements}

The grey level co-occurrence matrix (GLCM) has the ability to explore textural features and is often used to extract image textural features. Eight kinds of GLCM-based textures in three bands, red (R), green (G), and blue (B), of each digital image were calculated at deferent stages by using ENVI software, including

$$
V A R=\sum_{i} \sum_{j}(i-u)^{2} p(i, j)
$$




$$
\begin{gathered}
H O M=\sum_{i} \sum_{j} \frac{1}{1+(I-J)^{2}} p(i, j),(2) \\
C O N=\sum_{n=0}^{N_{g}-1} n^{2}\left\{\sum_{i=1}^{N_{g}} \sum_{j=1}^{N_{g}} p(i, j),|i-j|=n\right\} \text {, (3) } \\
E N T=-\sum_{i} \sum_{j} p(i, j) \log (p(i, j)),(4) \\
S E M=\sum_{i} \sum_{j}\{p(i, j)\}^{2},(5) \\
C O R=\frac{\sum_{i} \sum_{j}(i, j) p(i, j)-u_{x} u_{y}}{\sigma_{X} \sigma_{y}},(6)
\end{gathered}
$$

$M E A N=\frac{\sum_{i} \sum_{j} p(i, j)}{i j}$,

(7)

$$
D I S=\sum_{n=1}^{N_{g}-1} n\left\{\sum_{i=1}^{N_{g}} \sum_{j=1}^{N_{g}} p(i, j),|i-j|=n\right\}
$$

8

Where $p(i, j)$ : (i,j)th entry in a normalized gray-tone spatial-dependence matrix, $=P(i, j) / \mathrm{R}$. Ng: Number of distinct gray levels in the quantized image. $U_{x}, U_{y}, \sigma_{x}$ and $\sigma_{y}$ are the means and standard deviations. further details of the calculation are in available in [23] .

In this paper, a minimum window size of $3 \times 3$ pixels was used for textural feature extraction in order to reduce the computational complexity. In addition, the NDTI was used to explore its ability to predict AGB in winter wheat [7], which was defined as Equation (1):

$N D T I=(\mathrm{Ti}-\mathrm{Tj}) /(\mathrm{Ti}+\mathrm{Tj}) \quad($ Equation 1.)

where $T i$ and $T j$ represent two different randomly selected textures.

\section{Regression Modelling Methods Simple Linear Regression (LR)}

Linear regression (LR) has a simple and easily understandable model structure, which has great applicability in practical application [24]. The AGB estimation of winter wheat based on an LR model was used to evaluate the prediction performance of EWs, VIs, Texture, and NDTI for estimating AGB. The LR models were defend as shown in Equation (2):

$A G B=b X+a($ Equation 2.)

where $x$ represents the single input predictor variable, while $b$ and a represent the slope and intercept of the fitted line of the LR model, respectively.

\section{PLS and Random Forest (RF)}

Partial Least Squares regression is a dimension reduction method, which is a popular modelling approach frequently used in studies of vegetation because it provides an efficient way to make full use of spectral information $[25,26]$. Random forest (RF) is a powerful machine learning algorithm. Each tree of RF is constructed with a deterministic algorithm by selecting a group of random variables and a random sample from a calibration dataset. It can not only deal with a large number of input variables, but also uses a small number of variables to obtain a reasonable amount of prediction accuracy. In addition, RF regression is beneficial to overcoming the over-fitting problem of simple decision trees [27].

\section{Statistical Analysis}


In order to improve the applicability of the biomass estimation model, this paper analysed the relationship between AGB and different types of variables (effective wavelength, VIs, OTEXS, ONDTIS, as well as a combination of spectral indices and image indices). The flowchart in Figure 3 illustrates the experimental method. In this study, a total of 200 experimental data points were collected and randomly divided into a training set and a test set, with $2 / 3$ of the samples selected as a training dataset and $1 / 3$ samples as a test dataset. Three regression models (LR, PLS, RF) were established to estimate winter wheat biomass. The LR model was used to analyse the correlation between each calculated index and the AGB of winter wheat. The combination features were used as the input of the PLS and RF regression models to estimate the AGB of winter wheat. The AGB estimation performance of the three regression models was evaluated by the determination coefficient $\left(R^{2}\right.$, Equation (3)) and the root mean square error (RMSE, Equation (4)). Python software was used for all statistical analysis.

$\boldsymbol{R}^{2}=\mathbf{1}-\frac{\sum_{i=1}^{n}\left(y_{i}-\widehat{y}_{i}\right)^{2}}{\sum_{i=1}^{n}\left(y_{i}-\bar{y}_{\boldsymbol{t}}\right)^{2}}$ (Equation 3.)

$R M S E=\sqrt{\frac{1}{n} \sum_{i=1}^{n}\left(y_{i}-\widehat{y}_{\imath}\right)^{2}}$

(Equation 4.)

\section{Declarations}

\section{Acknowledgments}

We would like to thank Dongzhi Wang, Zhaojie Chu, Kaixun Han, Xujin Hu, and Hecai Yuan from Anhui University, for their fieldwork and contribution in data collection.

\section{Author Contributions}

L.Z., J.T., S.W. and Q.B. conceived and designed the experiments; L.Z. and S.W. performed the experiment; L.Z., J.T. analyzed the data and wrote the original manuscript; L.H., J.Z., S.W., Q.B., Y.Z. reviewed and revised the manuscript. All authors have read and agreed to the published version of the manuscript.

\section{Funding}

This research was funded by the National Natural Science Foundation of China (31701323), the Open Foundation of National Engineering Research Center for Agro-Ecological Big Data Analysis \& Application (AE202009) and the Natural Science Foundation of Anhui Province(2008085MF184).

\section{Availability of data and materials}

The datasets used and/or analyzed during the current study are available from the corresponding author on reasonable request.

\section{Ethics approval and consent to participate}

All authors read and approved the manuscript.

\section{Consent for publication}

All authors agreed to publish this manuscript.

\section{Competing interests}

The authors declare that they have no competing interests.

\section{References}

1. Khaki, S.; Wang, L.; Archontoulis, S.V. A CNN-RNN Framework for Crop Yield Prediction. Frontiers in Plant Science 2020, 10, 1750 - 
2. Luo, S.; Wang, C.; Xi, X.; Nie, S.; Fan, X.; Chen, H.; Yang, X.; Peng, D.; Lin, Y.; Zhou, G. Combining hyperspectral imagery and LiDAR pseudo-waveform for predicting crop LAl, canopy height and above-ground biomass. Ecological Indicators 2019, 102, 801-812, doi:10.1016/j.ecolind.2019.03.011.

3. Han, L.; Yang, G.; Dai, H.; Xu, B.; Yang, H.; Feng, H.; Li, Z.; Yang, X. Modeling maize above-ground biomass based on machine learning approaches using UAV remote-sensing data. Plant Methods 2019, 15, doi:10.1186/s13007-019-0394-z.

4. Jia, M.; Li, W.; Wang, K.; Zhou, C.; Yao, X. A newly developed method to extract the optimal hyperspectral feature for monitoring leaf biomass in wheat. Computers and Electronics in Agriculture 2019, 165, 104942.

5. Duan, D.D.; Zhao, C.J.; Li, Z.H.; Yang, G.J.; Zhao, Y.; Qiao, X.J.; Zhang, Y.H.; Zhang, L.X.; Yang, W.D. Estimating total leaf nitrogen concentration in winter wheat by canopy hyperspectral data and nitrogen vertical distribution. J. Integr. Agric. 2019, 18, 15621570, doi:10.1016/s2095-3119(19)62686-9.

6. Deery, D.M.; Rebetzke, G.J.; Jimenez-Berni, J.A.; Condon, A.G.; Smith, D.J.; Bechaz, K.M.; Bovill, W.D. Ground-Based LiDAR Improves Phenotypic Repeatability of Above-Ground Biomass and Crop Growth Rate in Wheat. Plant phenomics (Washington, D.C.) 2020, 2020, 8329798-8329798, doi:10.34133/2020/8329798.

7. Zheng, H.; Cheng, T.; Zhou, M.; Li, D.; Yao, X.; Tian, Y.; Cao, W.; Zhu, Y. Improved estimation of rice aboveground biomass combining textural and spectral analysis of UAV imagery. Precision Agriculture 2019, 20, 611-629, doi:10.1007/s11119-018-9600-7.

8. Walter, J.; Edwards, J.; McDonald, G.; Kuchel, H. Photogrammetry for the estimation of wheat biomass and harvest index. Field Crops Research 2018, 216, 165-174, doi:10.1016/j.fcr.2017.11.024.

9. Tan, C.; Zhang, Q.; Zhou, J.; Du, Y.; Wang, D.; Luo, M.; Zhang, H.; Guo, W. Remotely assessing above-ground fresh biomass weight of wheat based on the combinations of pair vegetation indexes from HJ-CCD images. Cluster Computing 2018.

10. El-Hendawy, S.; Elsayed, S.; Al-Suhaibani, N.; Alotaibi, M.; Hassan, W. Use of Hyperspectral Reflectance Sensing for Assessing Growth and Chlorophyll Content of Spring Wheat Grown under Simulated Saline Field Conditions. Plants $2021,10$.

11. Lin, L.; Yu, K.; Yao, X.; Deng, Y.; Liu, J. UAV Based Estimation of Forest Leaf Area Index (LAl) through Oblique Photogrammetry. Remote Sens. 2021, 13, 803.

12. Jla, B.; Dc, A.; Cga, B.; Zza, B.; Yxa, B.; Tha, B. Combining plant height, canopy coverage and vegetation index from UAV-based RGB images to estimate leaf nitrogen concentration of summer maize. Biosystems Engineering 2021, 202, 42-54.

13. Astaoui, G.; Dadaiss, J.E.; Sebari, I.; Benmansour, S.; Mohamed, E. Mapping Wheat Dry Matter and Nitrogen Content Dynamics and Estimation of Wheat Yield Using UAV Multispectral Imagery Machine Learning and a Variety-Based Approach: Case Study of Morocco. 2021.

14. Cai, W.; Zhao, S.; Wang, Y.; Peng, F.; Duan, Z. Estimation of Winter Wheat Residue Coverage Using Optical and SAR Remote Sensing Images. Remote Sens. 2019, 11, 1163-.

15. Chen, Y.; Dong, Y.; Ma, X.; Li, J.; Zhang, J. Fast Determination of the Rubber Content in Taraxacum Kok-Saghyz Fresh Biomass Using Portable Near Infrared Spectroscopy and Pyrolysis-Gas Chromatography. 2021.

16. Masowski, M.; Miedzianowska, J.; Delekta, M.; Czylkowska, A.; Strzelec, K. Natural Rubber Biocomposites Filled with Phyto-Ashes Rich in Biogenic Silica Obtained from Wheat Straw and Field Horsetail. Polymers 2021, 13, 1177.

17. Wahlang, R.; Chaturvedi, S.S. Relationship Between Above-Ground Biomass and Different Vegetation Indices of Forests of Ri-Bhoi District, Meghalaya, India. International Journal of Engineering and Technical Research 2020, V9.

18. Sun, Q.; Jiao, Q.; Qian, X.; Liu, L.; Dai, H. Improving the Retrieval of Crop Canopy Chlorophyll Content Using Vegetation Index Combinations. Remote Sens. 2021, 13, 470.

19. Triantafyllou, A.; Watlet, A.; Mouelic, S.L.; Camelbeeck, T.; Civet, F.; Kaufmann, O.; Quinif, Y.; Vandycke, S. 3-D digital outcrop model for analysis of brittle deformation and lithological mapping (Lorette cave, Belgium). Journal of structural geology 2019, 120, 5566.

20. Putra, B.; Soni, P. Improving nitrogen assessment with an RGB camera across uncertain natural light from above-canopy measurements. Precision Agriculture 2019.

21. Jiang, J.; Cai, W.; Zheng, H.; Cheng, T.; Yao, X. Using Digital Cameras on an Unmanned Aerial Vehicle to Derive Optimum Color Vegetation Indices for Leaf Nitrogen Concentration Monitoring in Winter Wheat. Remote Sens. 2019, $11,2667$.

22. Ma, J.; Liu, H.; Zheng, F.; Du, K.; Zhang, L.; Hu, X.; Sun, Z. Estimating growth related traits of winter wheat at seedling stages based on RGB images and convolutional neural network. Transactions of the Chinese Society of Agricultural Engineering 2019.

23. Haralick, R.M.; Shanmugam, K.; Dinstein, I.H. Texture Features for Image Classification. 1975.

Page 13/22 
24. Li, B.; Xu, X.; Zhang, L.; Han, J.; Jin, L. Above-ground biomass estimation and yield prediction in potato by using UAV-based RGB and hyperspectral imaging. ISPRS Journal of Photogrammetry and Remote Sensing 2020, 162, 161-172.

25. Yue, J.; Feng, H.; Yang, G.; Li, Z. A Comparison of Regression Techniques for Estimation of Above-Ground Winter Wheat Biomass Using Near-Surface Spectroscopy. Remote Sens. 2018, 10, 66.

26. Guo, J.; Zhang, J.; Xiong, S.; Zhang, Z.; Wei, Q.; Zhang, W.; Feng, W.; Ma, X. Hyperspectral assessment of leaf nitrogen accumulation for winter wheat using different regression modeling. Precision Agriculture 2021, 1-25.

27. Lu, N.; Zhou, J.; Han, Z.; Li, D.; Cao, Q.; Yao, X.; Tian, Y.; Zhu, Y.; Cao, W.; Cheng, T. Improved estimation of aboveground biomass in wheat from RGB imagery and point cloud data acquired with a low-cost unmanned aerial vehicle system. Plant Methods 2019, 15.

28. Jeon, H.; Choi, I.; Kweon, S.J.; Je, M. A Power-Efficient Radiation Sensor Interface with a Peak-Triggered Sampling Scheme for Mobile Dosimeters. Sensors 2020, 20, 3255.

29. Persson, H.J.; Jonzén, J.; Nilsson, M. Combining TanDEM-X and Sentinel-2 for large-area species-wise prediction of forest biomass and volume. International Journal of Applied Earth Observation and Geoinformation 2021, 96, 102275.

30. Li, S.; Yuan, F.; Ata-Ui-Karim, S.T.; Zheng, H.; Cao, Q. Combining Color Indices and Textures of UAV-Based Digital Imagery for Rice LAl Estimation. Remote Sens. 2019, 11, 1763-.

31. Heidrich, L.; Bae, S.; Levick, S.; Seibold, S.; Müller, J. Heterogeneity-diversity relationships differ between and within trophic levels in temperate forests. Nature Ecology \& Evolution 2020, 4, 1-9.

32. Pei, X.; Hong, S.; Li, M.; Zheng, L. The research of using spectral data to measure crop growth. 2015.

33. Qing, X.U.; Chen, J.B.; Tian, B.B. A overview of research on ship hull optimization sensitivity analysis methods. 2019.

34. Baret, S.F. Optimization of soil-adjusted vegetation indices. Remote Sensing of Environment 1996.

35. Schnell, J.A. Monitoring the vernal advancement and retrogradation (greenwave effect) of natural vegetation. Nasa/gsfct Type Final Report 1974.

36. Datt; B. Visible/near infrared reflectance and chlorophyll content in Eucalyptus leaves. International Journal of Remote Sensing 2010, 20, 2741-2759.

37. Gitelson, A.A. Remote estimation of canopy chlorophyll content in crops. Geophysical Research Letters 2005.

38. Huete, A. A comparison of vegetation indices over a global set of TM images for EOS-MODIS. Remote Sensing of Environment 1997, 59, 440-451.

39. Roujean, J.L.; Breon, F.M. Estimating PAR absorbed by vegetation from bidirectional reflectance measurements. Remote Sensing of Environment 1995, 51, 375-384.

\section{Figures}



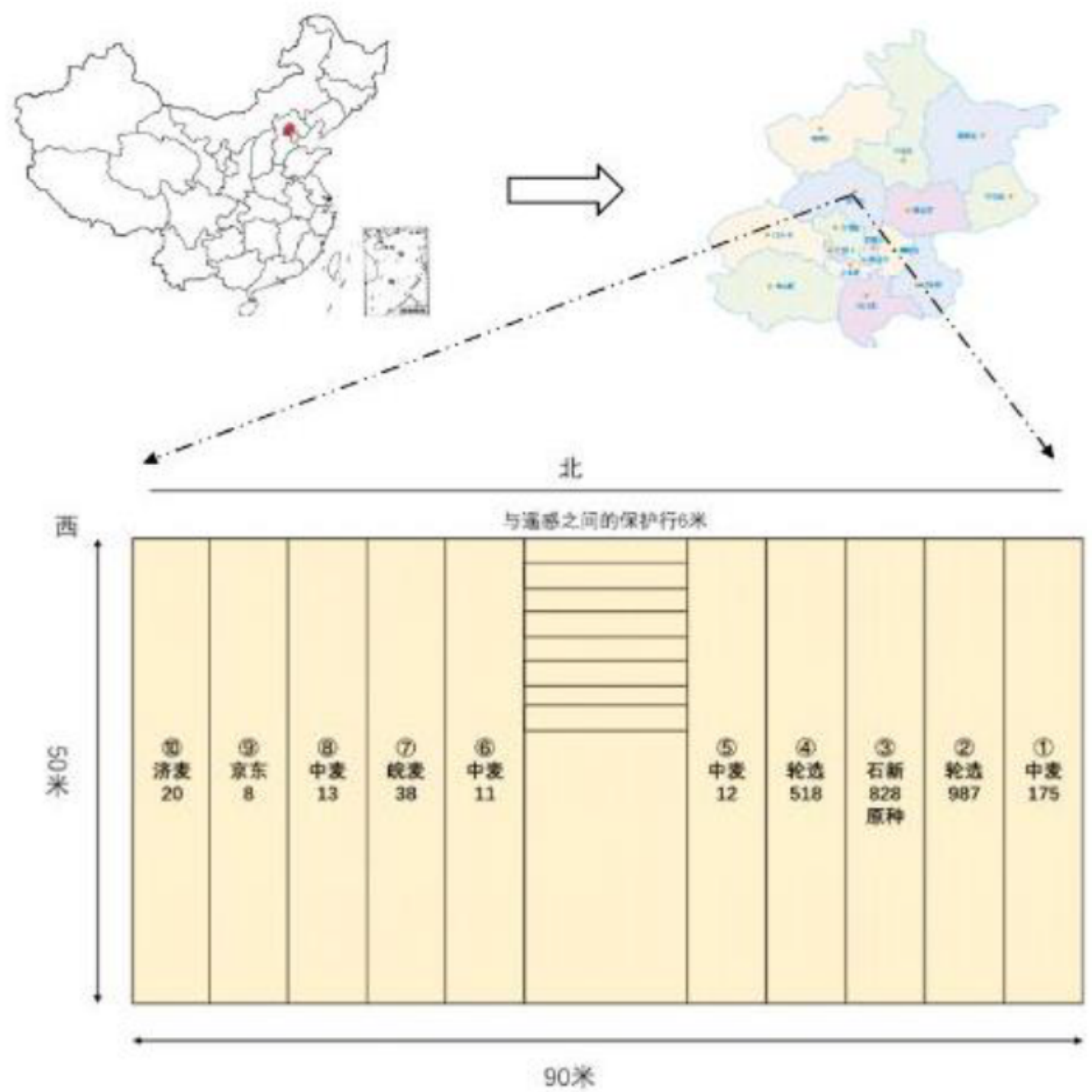

Figure 1

Experimental field 


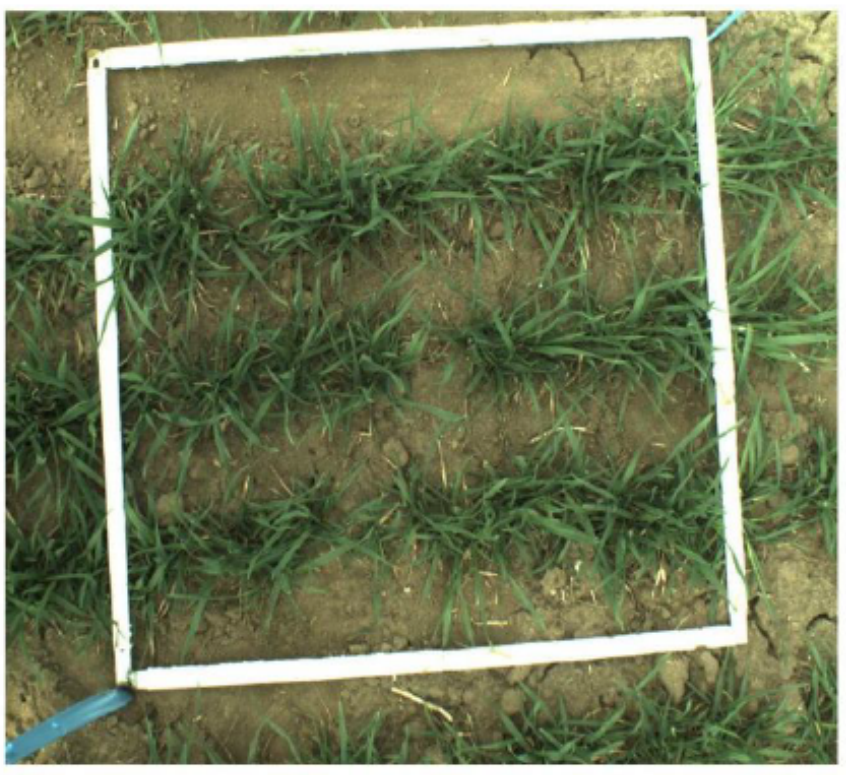

a

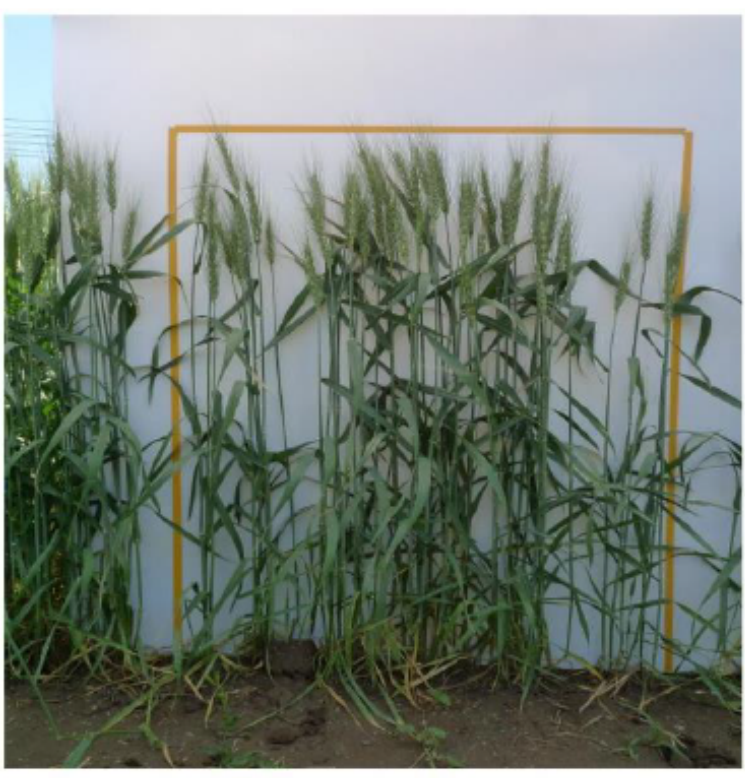

b

Figure 2

a,the canopy image at seedling stage;b,the side image at post-seeding stage.
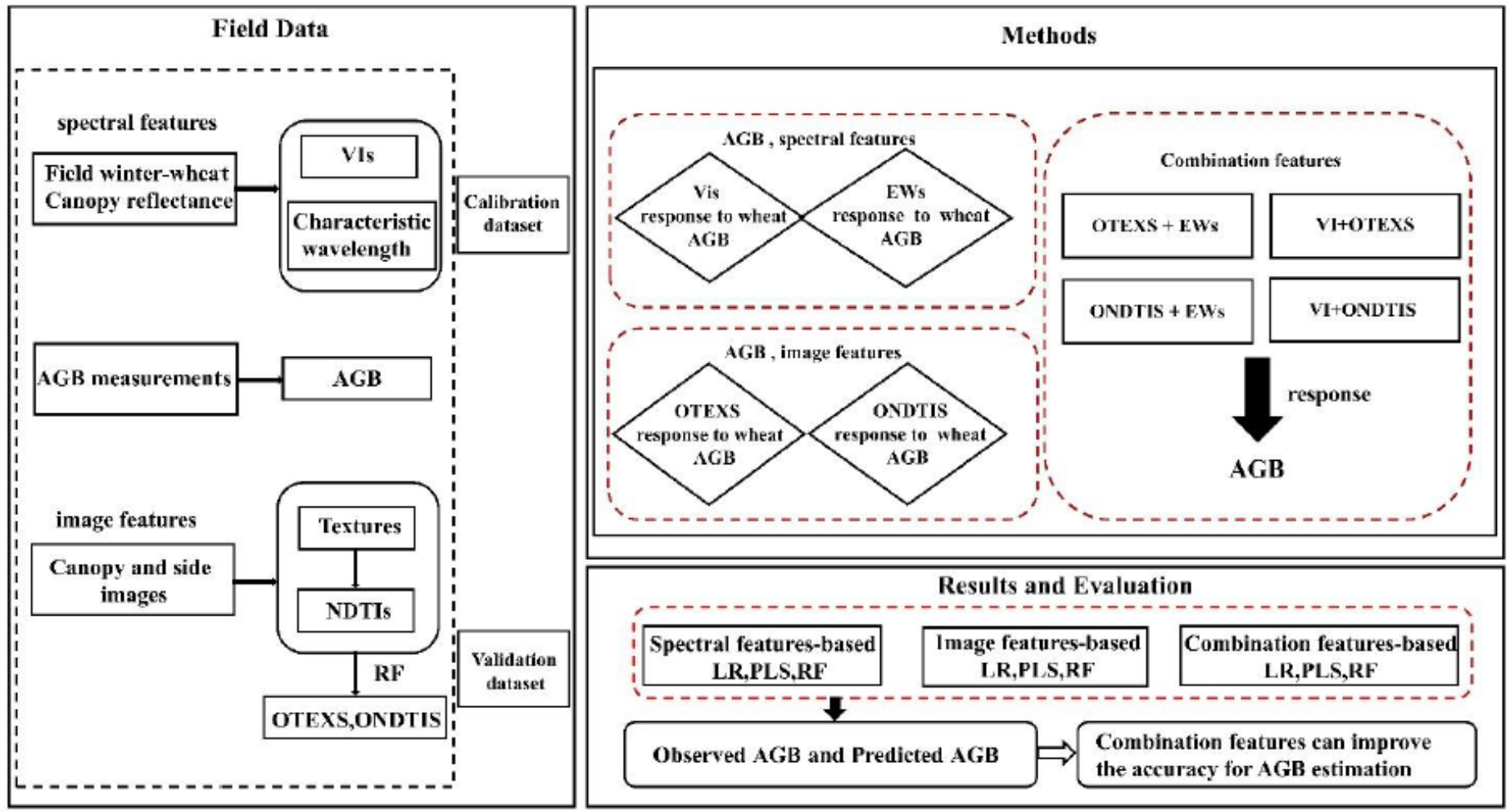

Results and Evaluation

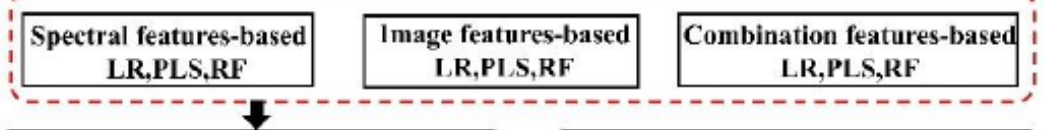

Observed AGB and Predicted AGB

Combination features can improve the accuracy for AGB estimation

\section{Figure 3}

Experiment methodology 

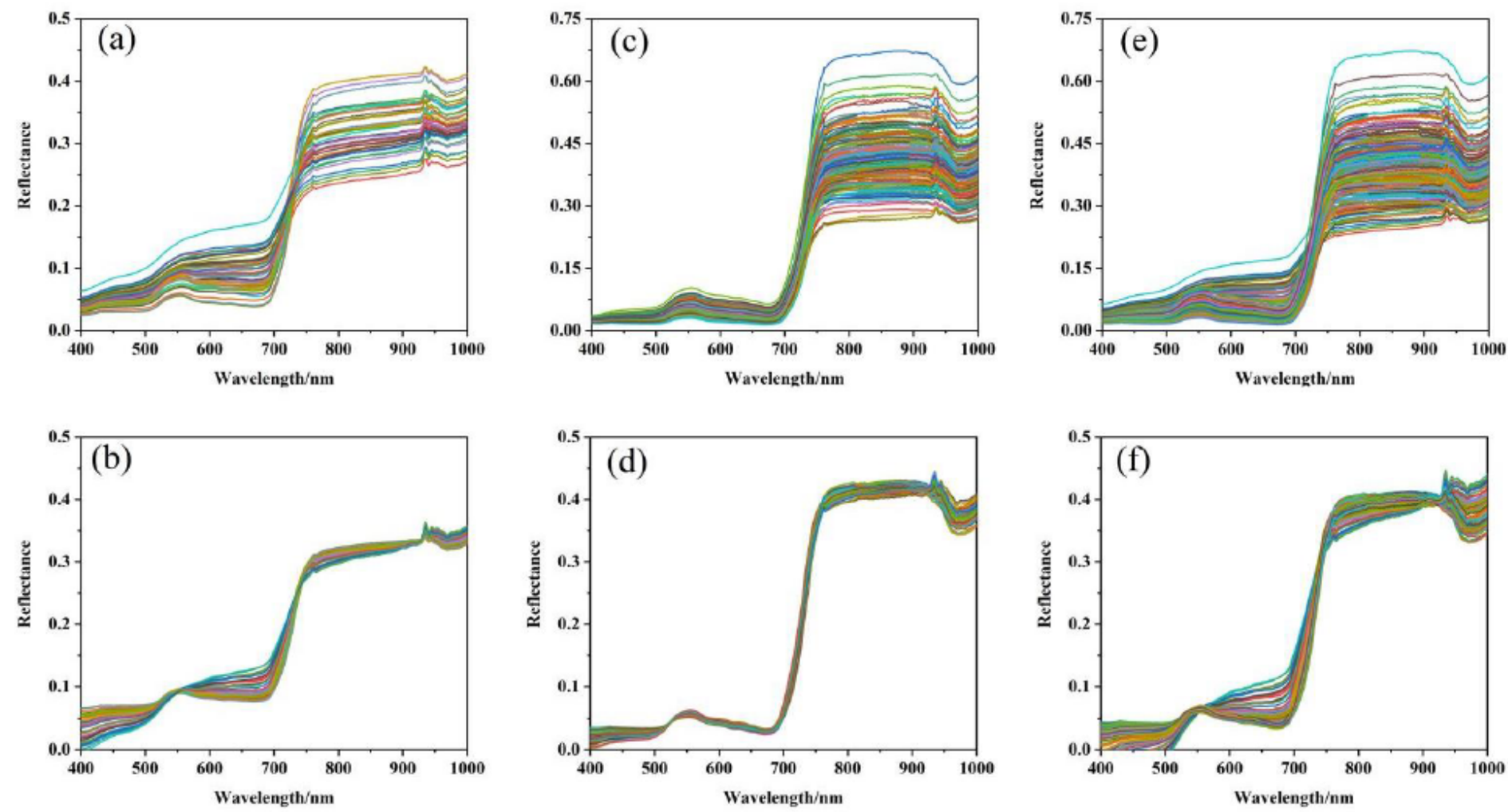

\section{Figure 4}

Reflectance spectra of the winter wheat canopy in different growth stages; Ori-Spectral in: (a) the seedling stage, (b) post-seedling stage, and (c) all stages; and Pre-Spectrum in (d) the seedling stage, (e) post-seedling stage and ( $\mathrm{f}$ ) all stages. 

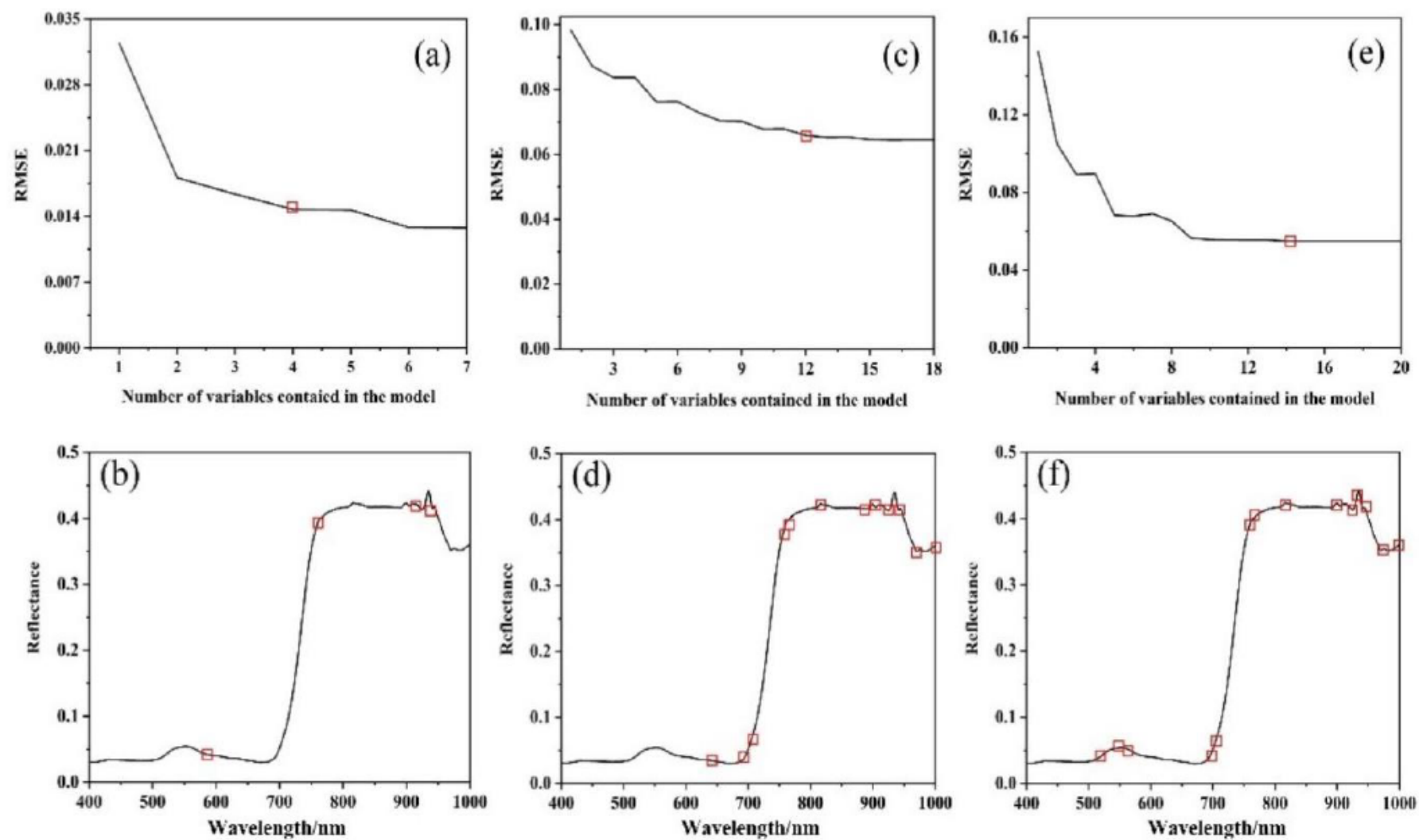

Figure 5. Reflectance spectra of the winter wheat canopy at seedling stage: (a), (b), (c) show the number of variables contained in the model in seedling stage, post-seedling stage, all stages, respectively; (d), (e), (f) show the wavelength in seedling stage, post-seedling stage, all stages, respectively. A square $(\square)$ represents the number of the effective wavelength.

\section{Figure 5}

See image above for figure legend.
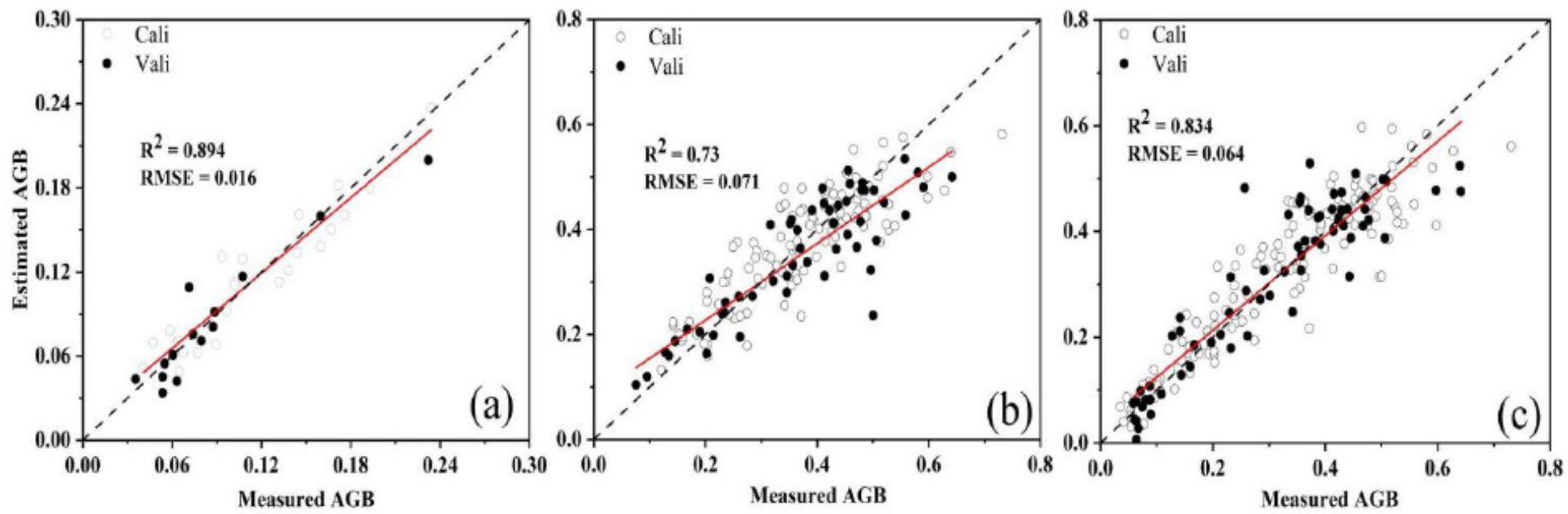

Figure 6

Validation of above-ground biomass (AGB) estimation models established by best performing: Characteristic wavelengths vs AGB for the following stages: (a) seedling, (b) post-seedling. 


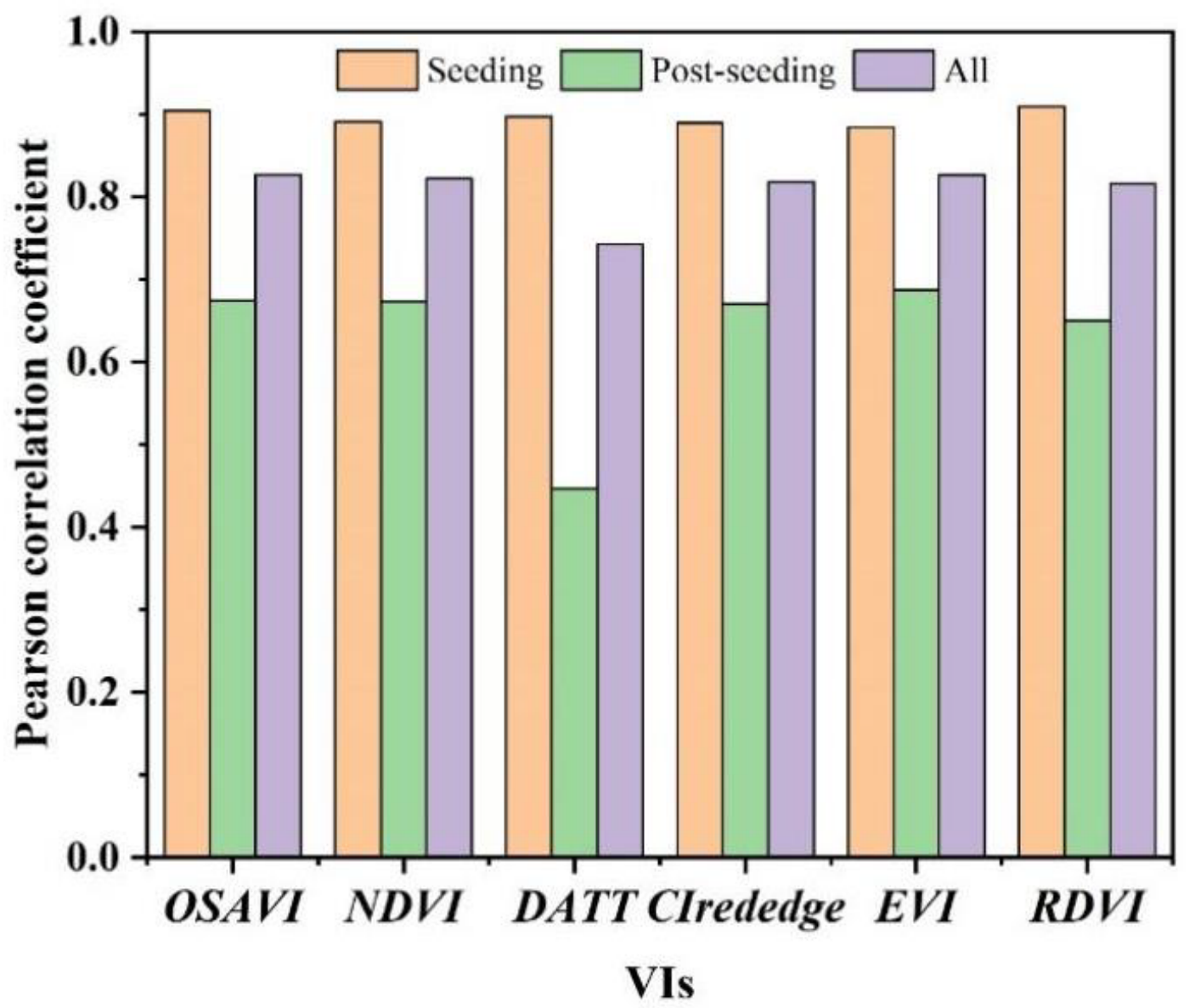

Figure 7

Pearson correlation coefficient between vegetation indices and above-ground biomass.
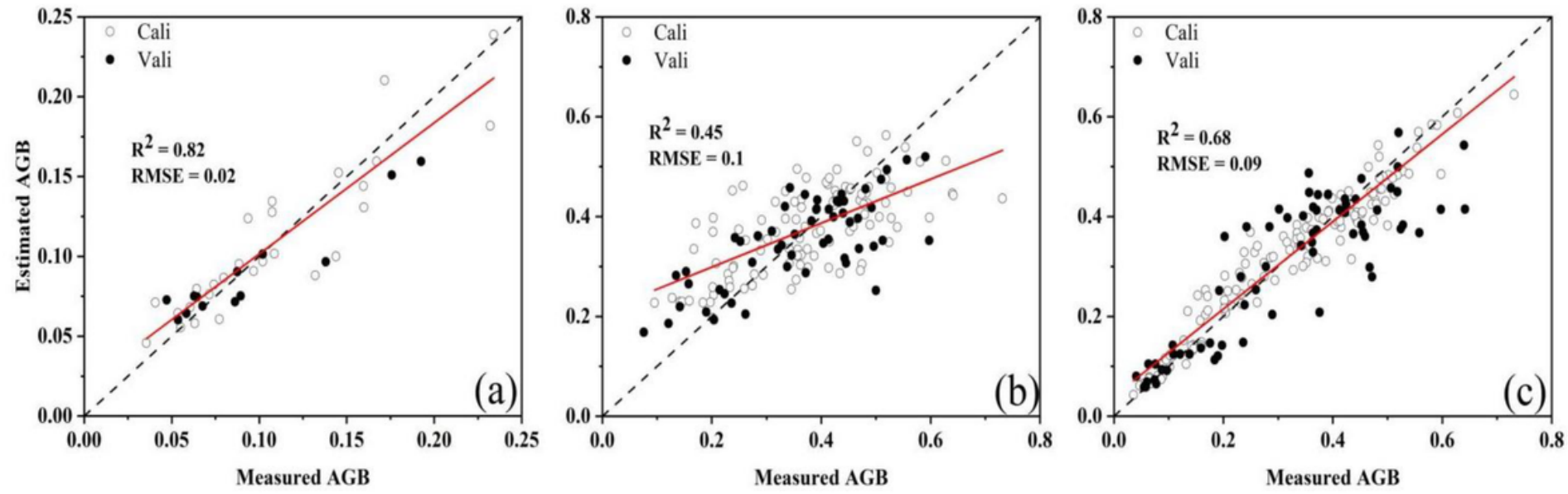

Figure 8

Validation of above-ground biomass (AGB) estimation models established by best performing: Vegetation index vs AGB for the following stages: (a) seedling, (b) post-seedling, (c) all stages 

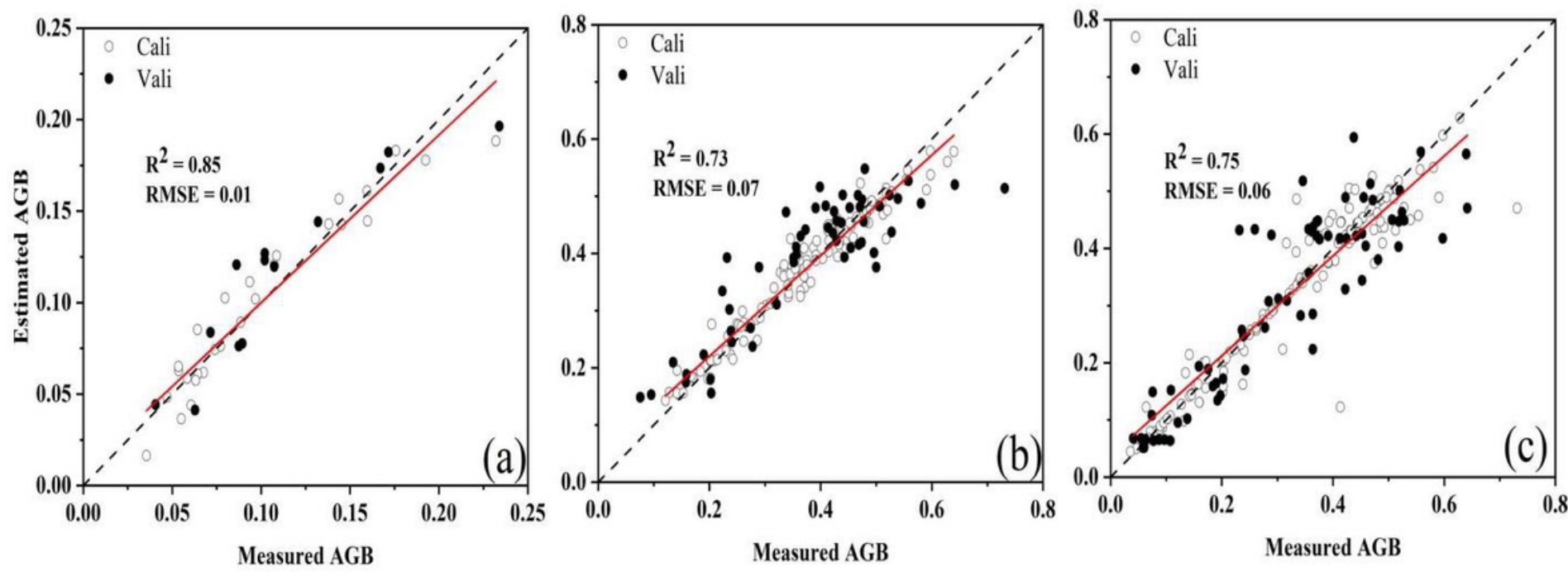

Figure 9

Validation of above-ground biomass (AGB) estimation models established by best performing: the optimal texture subset vs AGB for the following stages: (a) seedling, (b) post-seedling, (c) all stages

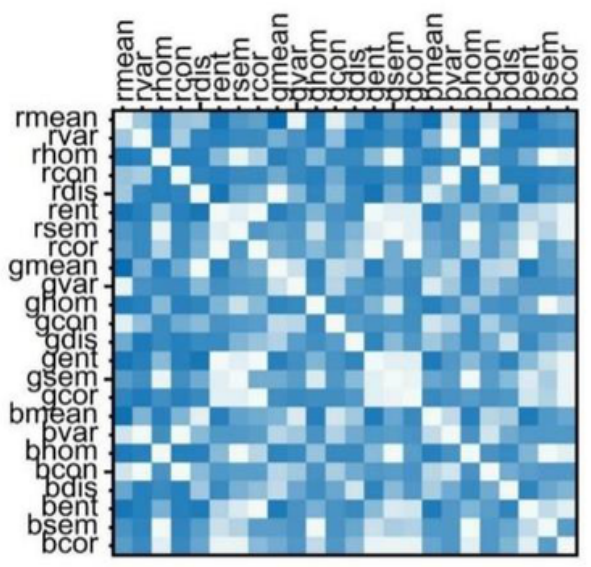

a

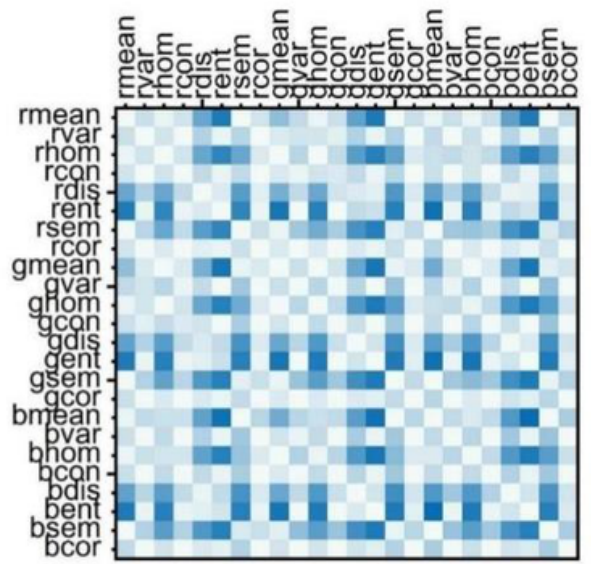

b

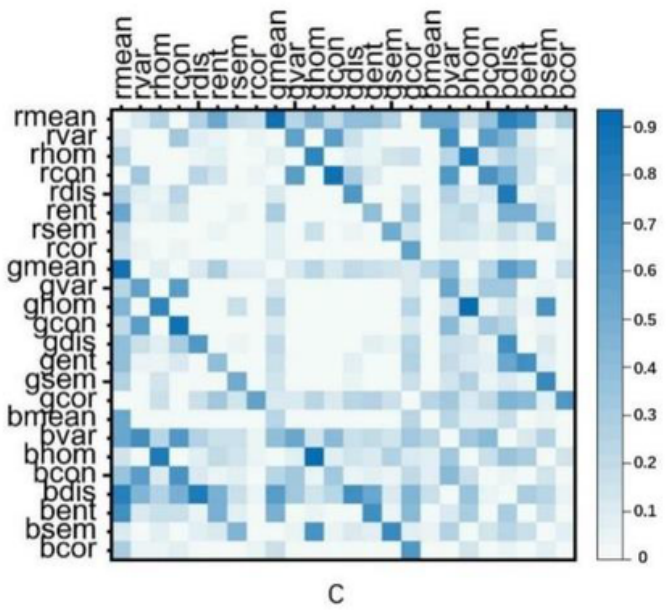

Figure 10

$\mathrm{R}^{2}$ of the normalized differential texture index-based linear regression models for estimating above-ground biomass at the (a) seedling stage, (b) post-seedling stage and (c) all stages. 

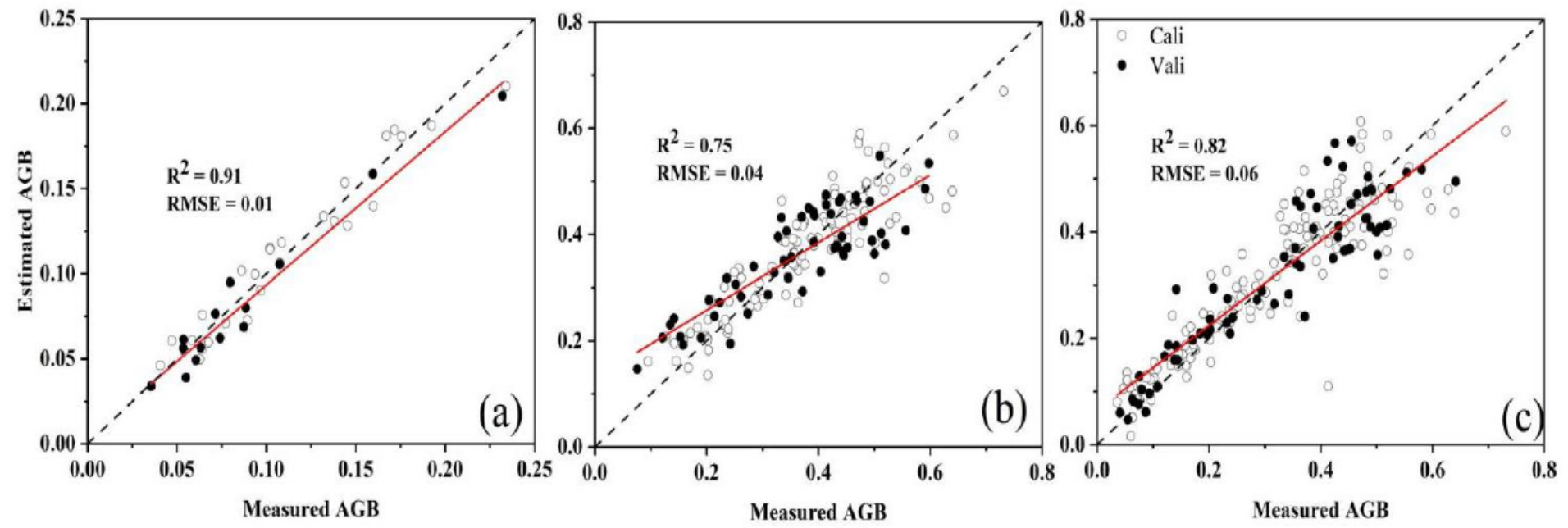

Figure 11

Validation of above-ground biomass (AGB) estimation models established by best performing : The optimal normalized difference texture index subset vs AGB for the following stages: (a) seedling, (b) post-seedling, (c) all stages.
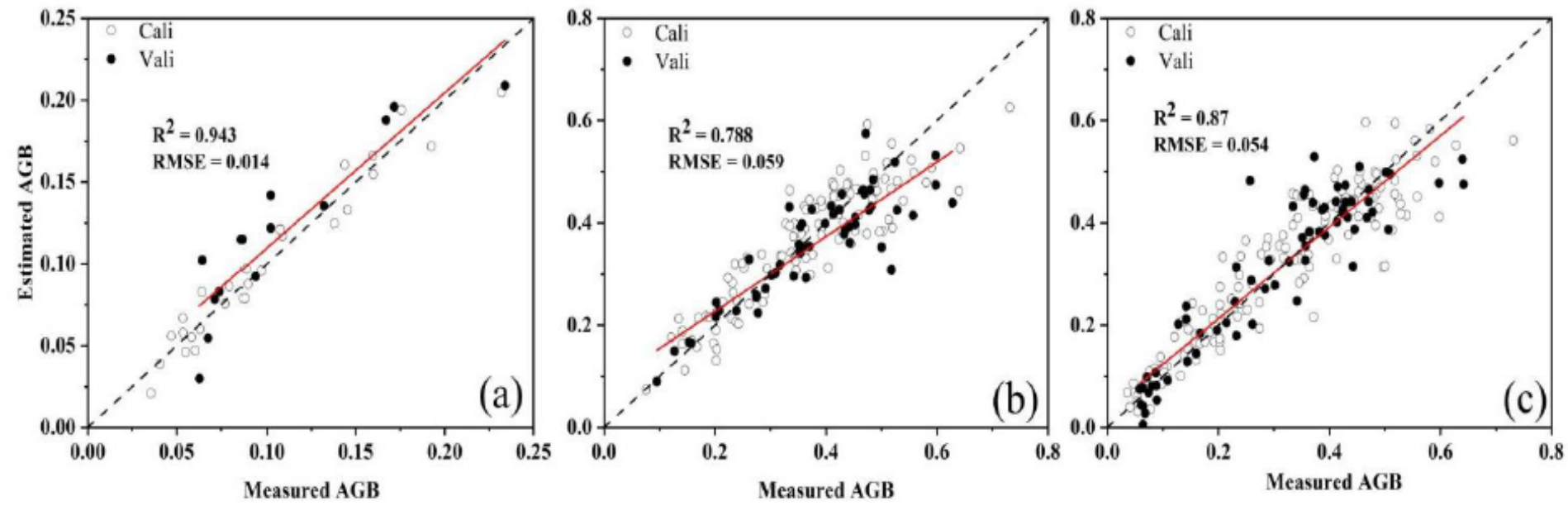

Figure 12

Validation of above-ground biomass (AGB) estimation models established by best performing : the optimal normalized difference texture index subset vs AGB for the following stages: (a) seedling, (b) post-seedling, (c) all stages. 

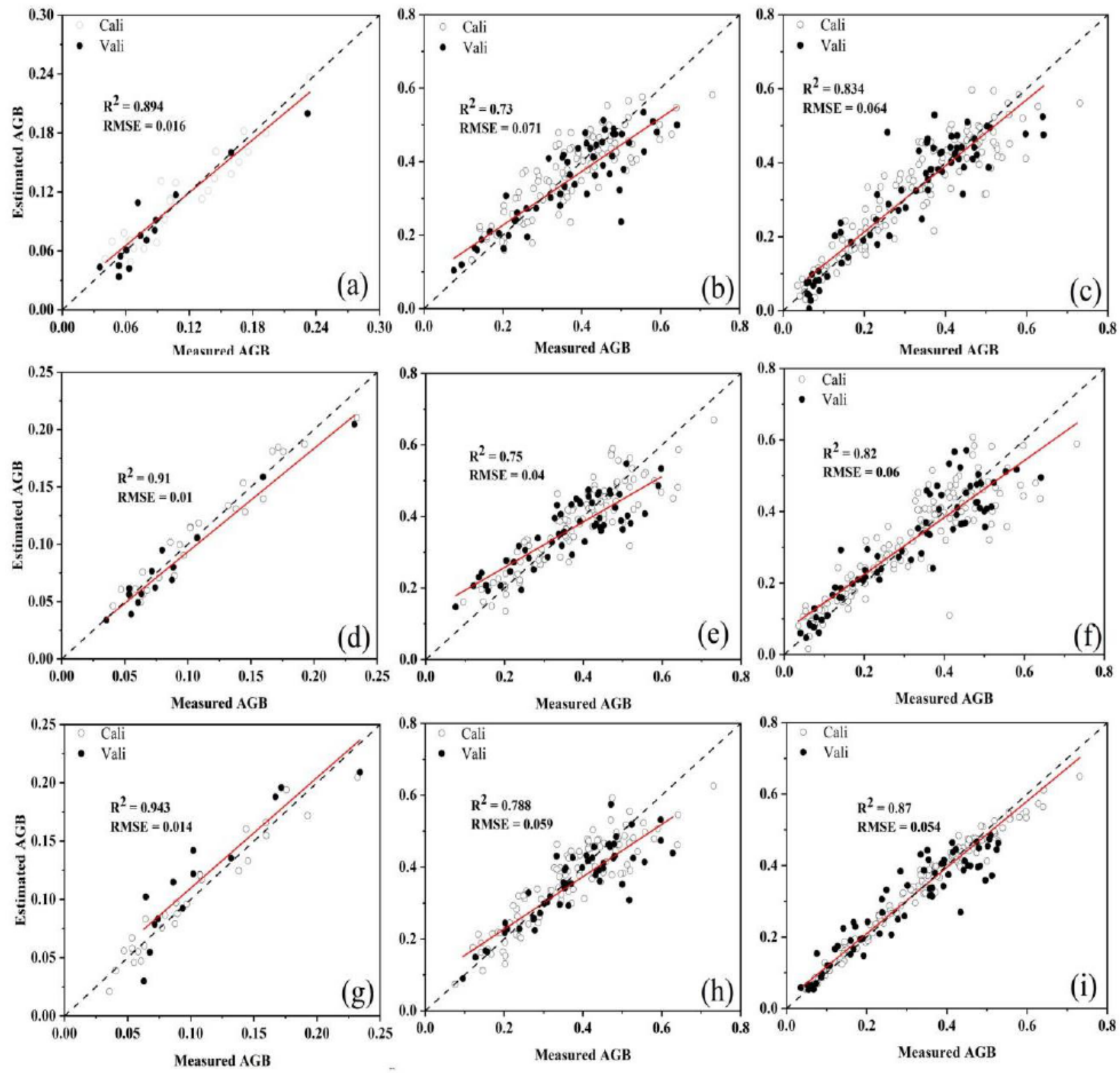

\section{Figure 13}

Validation of above-ground biomass (AGB) estimation models established by best performing spectral, image, and combination features: characteristic wavelengths for the following stages: (a) seedling, (b) post-seedling, (c) all stages; ONDTIS for these stages, (d) seedling, (e) post-seedling, (f) all stages; OTEX+ characteristic wavelengths for these stages, (g) seedling, (h) post-seedling and (i) all stages. 